\title{
New hot subdwarf variables from the EC survey
}

\author{
D. Kilkenny, ${ }^{1 \star}$ H. L. Worters ${ }^{2}$ and A. E. Lynas-Gray ${ }^{1,3,4}$ \\ ${ }^{1}$ Department of Physics and Astronomy, University of the Western Cape, Private Bag X17, Bellville 7535, South Africa. \\ ${ }^{2}$ South African Astronomical Observatory, Observatory 7935, South Africa \\ ${ }^{3}$ Department of Physics and Astronomy, University College London, Gower Street, London WC1E 6BT, England \\ ${ }^{4}$ Department of Physics, University of Oxford, Oxford OX1 3RH, England
}

Accepted 2019 February 27. Received 2019 February 27; in original form 2019 January 14

\begin{abstract}
We present new results for hot subdwarf stars from the Edinburgh-Cape (EC) blue object survey. EC 03089-6421, an sdO star recently discovered to be a very rapid pulsator with periods near 31.1 and $34.2 \mathrm{~s} \mathrm{(29.2} \mathrm{and} 32.1 \mathrm{mHz}$ ), is shown to have an even faster pulsation near $26.6 \mathrm{~s}(37.6 \mathrm{mHz})$ although all pulsations are variable in amplitude and effectively disappear in one of our runs. Five stars are discovered to be of the rapidly-pulsating $\left(\mathrm{sdBV}_{r}\right)$ type: EC 01441-6605, EC 10834-1301, EC 11275-2504, EC 11545-1459, and EC 212815010 each exhibit between one and three variations in the range $2-3 \mathrm{~min}(\sim 8.2-6.4 \mathrm{mHz})$ and are therefore p-mode pulsators. EC 15061-1442 shows a large-amplitude variation with a period $\sim 0.075 \mathrm{~d}$ if the star is a reflection effect binary and $0.15 \mathrm{~d}$ if an ellipsoidal variable. No eclipses are detected. Low-resolution EC survey classification spectroscopy is combined with published photometry from GALEX, WISE, and 2MASS and parallaxes from GAIA to derive, where possible, fundamental stellar parameters. The $\mathrm{sdBV}_{r}$ stars have $T_{\text {eff }}$ in the range $34000-45000 \mathrm{~K}$ and $\log g$ between 5.0 and 5.6 consistent with p-mode pulsators. Derived stellar masses and radii are in the ranges $0.15-0.4 \mathrm{M}_{\odot}$ and $0.15-0.2 R_{\odot}$. The sdOV star, EC $03089-6421$ is found to have $T_{\text {eff }}=52000 \pm 3000 \mathrm{~K}$ and $\log g=5.6 \pm 0.2$, and with a mass $\sim 0.1 \mathrm{M}_{\odot}$ could be a progenitor to an extremely low-mass white dwarf.
\end{abstract}

Key words: Stars: oscillations -Stars: variables.

\section{INTRODUCTION}

The main purpose of the recently completed Edinburgh-Cape (EC) survey was to provide a substantial sample of Southern hemisphere blue objects for further study (Stobie et al. 1997; Kilkenny et al. 1997b, 2015, 2016; O’Donoghue et al. 2013). Following the discoveries of the rapidly-pulsating sdB stars (Kilkenny et al. 1997a), the slowly-pulsating sdB stars (Green et al. 2003), the hybrid pulsators (Oreiro et al. 2004; Schuh et al. 2006), and the pulsating sdO stars (Woudt et al. 2006), we have continued to search, especially amongst the EC stars, for new examples. These relatively new classes of variable do not have large numbers - there is only one known Helium-rich sdB (HesdB) pulsator (Ahmad \& Jeffery 2005, Naslim et al. 2011) and the sdO variables are represented by just three cases in the general field (see Kilkenny, Worters \& $\emptyset$ stensen 2017) and a handful of stars in $\omega$ Centauri (Randall et al. 2011, Randall et al. 2016). And yet they are extremely useful for determining the structure of post-giant-branch stars via the identification of pulsation modes (Charpinet et al. 2008; Van Grootel et al. 2008, for example), determining the rate of evolution

\footnotetext{
^E-mail: dkilkenny@uwc.ac.za
}

via secular frequency changes (Lutz, Schuh \& Silvotti 2012) caused by stellar mean density changes, as well as detecting the existence of low-mass companions deduced from cyclic frequency changes caused by motion about the barycentre (Barlow et al. 2011). It has even been possible to detect large planetary bodies in this way (Silvotti et al. 2007).

Of course, the Kepler satellite mission (Borucki et al. 2010), originally designed to search for exoplanets, has substantially contributed to the study of all types of variable star - the superb data quality, the long, almost uninterrupted baseline, and the relatively high cadences of observation providing many new insights. In the hot subdwarf field, new discoveries include the detection of unusually long rotation periods (Pablo et al. 2012; see also reviews by Heber 2016 and Reed et al. 2018); the demonstration of stochasticity in the pulsations of an sdB star (Østensen et al. 2014); the discovery of orbital Doppler beaming in sdB + WD binaries (Telting et al. 2012); radial differential rotation in a hybrid pulsator (Foster et al. 2015); and even the inference of orbiting Earth-like planets (Charpinet et al. 2011). Even so, in the case of Kepler (for example) it was necessary to specify targets beforehand and so there is still a place for ground-based discovery. In this context, preparation of target lists for the Transiting Exoplanet Survey Satellite (TESS; Ricker et al. 2014, launched in 2018) led 
Table 1. EC photometry and spectral types for the stars discussed in this paper.

\begin{tabular}{lccccc}
\hline EC & $V$ & $B-V$ & $U-B$ & EC Type & Var \\
\hline $01441-6605$ & 14.84 & +0.02 & -0.98 & $\mathrm{sdB}+\mathrm{F}$ & $\mathrm{sdBV}_{r}$ \\
$03089-6421$ & 14.80 & -0.35 & -1.25 & $\mathrm{sdO}$ & $\mathrm{sdOV}$ \\
$10384-1301$ & 15.58 & -0.12 & -1.12 & sdOB & $\mathrm{sdBV}_{r}$ \\
$11275-2504$ & 14.06 & -0.28 & -1.06 & sdOB & $\mathrm{sdBV}_{r}$ \\
$11545-1459$ & 16.03 & -0.28 & -1.09 & $\mathrm{sdB}$ & $\mathrm{sdBV}_{r}$ \\
$15061-1442$ & 13.37 & -0.11 & -0.87 & sdB & binary \\
$21281-5010$ & 15.30 & -0.27 & -1.09 & sdOB & sdBV $_{r}$ \\
\hline
\end{tabular}

to the entirely fortuitous discovery of the very rapidly-pulsating sdO star, EC 03089-6421 (Kilkenny et al. 2017 - and discussed below).

The range in the nature of variability in the rapidly-pulsating hot subdwarfs is substantial, from singly periodic (Barlow et al. 2011) to stars with over 40 frequencies detected even from ground-based observations (Koen et al. 1998). With the advent of the Kepler satellite mission, hundreds of frequencies have been discovered in some of the slowly-pulsating and hybrid hot subdwarfs (Reed et al. 2014, for example). The pulsations are often quite variable in amplitude (Kilkenny 2010) and can even effectively disappear (Hutchens et al. 2017). In the context of this paper, when we say that amplitudes are variable, we are referring to the phenomenon rather than the cause. A pulsation might have a variable amplitude (for example, if it were damped) but, as the referee has pointed out, if we have simply not resolved two or more frequencies in a narrow range which then produce beating, it would appear as a single frequency with (apparently) variable amplitude. Kepler has shown the latter for many sdBV stars, especially the slow-pulsators; Reed et al. (2014) and Kern et al. (2017) are examples of the rich frequency spectra revealed.

In this paper, we report the discovery of five new, rapidlypulsating $\left(\mathrm{sdBV}_{r}\right)$ stars and a reflection effect (but non-eclipsing) binary. We also report a new very high frequency variation in the known rapidly pulsating sdO star, EC 03089-6421. Table 1 lists the existing EC $U B V$ photometry and spectral types for the stars discussed here.

The referee has noted that we have employed an unusual observing tactic in making a number of short runs on each target; in fact, this was unintentional. We had originally planned a short 'discovery' paper to be submitted in mid-2017; this was delayed. We discovered another variable (EC 01441-6605) in late 2017 and then made the decision to add spectroscopic analysis to the paper which took further time. We never planned long campaigns for any of the targets because of the (then) approaching launch date for TESS which at that time entertained the possibility of a $20 \mathrm{~s}$ cadence (which may still occur). In the meantime we had obtained some further data, results from which it seemed sensible to publish with the early material. These new $\mathrm{sdBV}_{r}$ pulsators are included in the TESS target lists.

\section{OBSERVATIONS AND REDUCTIONS}

The photometry described in this paper was obtained at the Sutherland site of the South African Astronomical Observatory (SAAO) using either the new $1 \mathrm{~m} \mathrm{Lesedi}^{1}$ telescope (currently being commissioned) with the Sutherland High-Speed Optical Camera
(SHOC) or the $1 \mathrm{~m}$ Elizabeth $^{2}$ telescope with the STE3 CCD photometer.

The STE3 CCD is a $512 \times 512$ detector, which has a read-out time of about $6 \mathrm{~s}$ when pre-binned $2 \times 2$, the mode used for all our STE3 observations, which were made without a filter to maximize counts. Reduction of the CCD frames and magnitude extraction were performed using software written by Darragh O'Donoghue and based on the DOPHOT program described by Schechter, Mateo \& Saha (1993).

The SHOC instrument uses an Andor E2V $1024 \times 1024$ CCD and works in frame-transfer mode so that the read-out time is effectively zero. It has been designed to run at up to 20 frames $\mathrm{s}^{-1}$ with highaccuracy timing for each frame, though we have used it at much lower sampling rates. Full details of the system can be found in Coppejans et al. (2013). The SHOC data were reduced using an inhouse SAAO aperture photometry pipeline based on IRAF routines and again, no filter was used.

Where possible, we have differentially corrected target star data using non-variable stars on the CCD frames. The STE3 CCD on the $1 \mathrm{~m}$ telescope has only a small field of view (about $2 \times 2$ arcmin), whereas the SHOC camera on the new $1 \mathrm{~m}$ telescope has a field of view of $5.7 \times 5.7$ arcmin so that it was generally much easier to find suitable comparison stars on the latter. Where no suitable comparisons existed, we have retained data only from photometric nights and have removed the effect of atmospheric extinction using a quadratic approximation. In relatively short observing sequences therefore we cannot distinguish lower frequency variations in stars from residual atmospheric variation.

The frequency analyses described below were carried out with Darragh O'Donoghue's EAGLE program which uses the Fourier transform method of Deeming (1975) as modified by Kurtz (1985). A periodogram was calculated for each light curve, the highest peak removed by a least-squares fitted sinusoid (Deeming 1968) and the periodogram recalculated, and so on.

Since the cycle times (including read-out) for the STE3 instrument were typically around 20-25 s (Table 2) and, since sdBV pulsation times are usually more than $\sim 2 \mathrm{~min}$, the periodograms displayed in the various figures mostly cover the range $0-20 \mathrm{mHz}$ - that is, down to $50 \mathrm{~s}$ (equivalent to the Nyquist frequency). Where SHOC data were obtained, usually at much higher cadences, the periodograms for these have been checked to the Nyquist frequency in each case, but are only displayed to the same $20 \mathrm{mHz}$ as the STE3 data, except in the case of the very short period EC 03089-6421 (Fig. 1).

\section{THE SDOV STAR, EC 03089-6421}

EC 03089-6421 was classified sdO in Zone 3 of the EC survey (Kilkenny et al. 2015), but was picked up much earlier in the BPS survey (BPS CS 31064-0017; Beers, Preston \& Schectman 1992) with photometry $V=14.75,(B-V)=-0.34$, and $(U-B)=$ -1.18 by Norris, Ryan \& Beers (1999; cf Table 1) but with no spectral type assigned. Kilkenny, Worters \& Østensen (2017) found the star to be variable with extremely short periods of 34.2 and $31.1 \mathrm{~s}$ (frequencies of 29.2 and $32.1 \mathrm{mHz}$, respectively). They gave sample light curves for EC 03089-6421 and noted that the pulsation amplitudes appeared to be variable on rather short time-scales declining visibly during the course of a single night and appearing to decline and recover more slowly over several nights. 
Table 2. Observing log and extracted frequencies, amplitudes, and periods for the known sdO pulsator EC 03089-6421 and the five new sdB/sdOB pulsators. $t_{\text {exp }}$ is the frame exposure time, $\mathrm{n}$ is the number of CCD frames. There is no read-out time with SHOC but the STE3 read-out takes $\sim 6 \mathrm{~s}$, so that the total time per observation is $t_{\exp }+6 \mathrm{~s}$. Numbers in parentheses are the formal errors in the last digit(s) of the frequencies from the least-squares fitting procedure. Typical formal errors in the amplitudes are $\sim 0.0005-0.001 \mathrm{mag}$.

\begin{tabular}{|c|c|c|c|c|c|c|c|c|c|c|c|c|c|}
\hline Star/Date & Phot & $\begin{array}{r}t_{\exp } \\
(\mathrm{s})\end{array}$ & $\mathrm{n}$ & $\begin{array}{l}\text { Run } \\
\text { (hr) }\end{array}$ & $\begin{array}{l}\text { Freq. } \\
(\mathrm{mHz})\end{array}$ & $\begin{array}{l}\text { Ampl. } \\
\text { (mag) }\end{array}$ & $\begin{array}{l}P \\
(s)\end{array}$ & $\begin{array}{l}\text { Freq. } \\
(\mathrm{mHz})\end{array}$ & $\begin{array}{l}\text { Ampl. } \\
\text { (mag) }\end{array}$ & $\begin{array}{l}P \\
(s)\end{array}$ & $\begin{array}{l}\text { Freq. } \\
(\mathrm{mHz})\end{array}$ & $\begin{array}{c}\text { Ampl. } \\
\text { (mag) }\end{array}$ & $\begin{array}{l}P \\
(s)\end{array}$ \\
\hline \multicolumn{14}{|l|}{ EC 03089-6421 } \\
\hline 2017 Jan 14/15a & SHOC & 2 & 1800 & 1.0 & $32.13(2)$ & 0.012 & 31.1 & $29.24(2)$ & 0.013 & 34.2 & $37.61(3)$ & 0.007 & 26.6 \\
\hline $14 / 15 b$ & SHOC & 2 & 1800 & 1.0 & $32.14(1)$ & 0.010 & 31.1 & $29.25(1)$ & 0.010 & 34.2 & $37.64(1)$ & 0.005 & 26.6 \\
\hline $16 / 17$ & SHOC & 2 & 2357 & 1.3 & $31.98(5)$ & 0.004 & 31.3 & & & & & & \\
\hline Oct $10 / 11$ & STE3 & 5 & 695 & 2.3 & $32.12(1)$ & 0.007 & 31.1 & $29.27(1)$ & 0.002 & 34.2 & $37.67(1)$ & 0.004 & 26.5 \\
\hline $11 / 12$ & STE3 & 5 & 797 & 2.5 & $32.13(1)$ & 0.006 & 31.1 & $29.37(3)$ & 0.002 & 34.1 & $37.65(2)$ & 0.003 & 26.6 \\
\hline $23 / 24$ & STE3 & 5 & 634 & 2.0 & $32.14(1)$ & 0.010 & 31.2 & $29.27(2)$ & 0.002 & 34.2 & $37.64(1)$ & 0.004 & 26.6 \\
\hline 2018 Feb 16/17 & STE3 & 5 & 408 & 1.3 & $32.14(1)$ & 0.018 & 31.1 & $29.26(1)$ & 0.017 & 34.2 & $37.72(3)$ & 0.003 & 26.5 \\
\hline $19 / 20$ & STE3 & 5 & 363 & 1.4 & $32.15(2)$ & 0.007 & 31.1 & $29.25(1)$ & 0.025 & 34.2 & $37.58(3)$ & 0.004 & 26.6 \\
\hline $20 / 21$ & STE3 & 5 & 450 & 1.2 & $32.13(2)$ & 0.005 & 31.1 & $29.25(1)$ & 0.025 & 34.2 & $37.65(2)$ & 0.006 & 26.6 \\
\hline \multicolumn{14}{|l|}{ EC 01441-6605 } \\
\hline 2017 Oct $18 / 19$ & STE3 & 10 & 298 & 1.4 & $7.038(18)$ & 0.005 & 142.1 & $6.862(24)$ & 0.003 & 145.7 & $8.133(25)$ & 0.003 & 122.9 \\
\hline $19 / 20$ & STE3 & 8 & 797 & 3.2 & $7.042(4)$ & 0.004 & 142.0 & $6.882(4)$ & 0.005 & 145.3 & $8.128(7)$ & 0.003 & 123.0 \\
\hline $20 / 21$ & STE3 & 10 & 658 & 3.0 & $7.044(6)$ & 0.005 & 142.0 & $6.869(7)$ & 0.004 & 145.6 & $8.121(10)$ & 0.002 & 123.1 \\
\hline $21 / 22$ & STE3 & 10 & 564 & 2.7 & $7.040(4)$ & 0.006 & 142.0 & $6.842(7)$ & 0.003 & 146.1 & $8.122(8)$ & 0.003 & 123.1 \\
\hline $23 / 24$ & STE3 & 10 & 554 & 2.5 & $7.042(5)$ & 0.006 & 142.0 & $6.873(8)$ & 0.004 & 145.5 & $8.124(8)$ & 0.004 & 123.1 \\
\hline \multicolumn{14}{|l|}{ EC 10384-1301 } \\
\hline 2017 May 21/22 & STE3 & 15 & 167 & 1.0 & $6.943(16)$ & 0.011 & 144.0 & & & & & & \\
\hline May $22 / 23$ & SHOC & 2.5 & 1193 & 0.8 & $6.994(16)$ & 0.010 & 143.0 & & & & $6.570(37)$ & 0.004 & 152.2 \\
\hline May 30/31 & SHOC & 3 & 1792 & 1.5 & $7.006(11)$ & 0.010 & 142.7 & $6.684(19)$ & 0.006 & 149.6 & & & \\
\hline 2018 Feb $14 / 15$ & STE3 & 15 & 374 & 2.2 & & & & $6.755(19)$ & 0.009 & 148.1 & & & \\
\hline Feb $15 / 16$ & STE3 & 15 & 383 & 2.3 & $6.986(12)$ & 0.005 & 143.1 & $6.764(7)$ & 0.008 & 147.8 & $6.568(19)$ & 0.003 & 152.2 \\
\hline \multicolumn{14}{|l|}{ EC 11275-2504 } \\
\hline 2016 Feb 09/10 & STE3 & 20 & 526 & 3.9 & $6.778(2)$ & 0.008 & 147.5 & $7.572(14)$ & 0.001 & 132.1 & & & \\
\hline 2017 Jan 14/15 & SHOC & 2 & 3542 & 2.0 & $6.778(2)$ & 0.009 & 147.5 & $7.813(17)$ & 0.001 & 128.0 & & & \\
\hline $17 / 18$ & SHOC & 1 & 2357 & 0.8 & $6.781(1)$ & 0.009 & 147.5 & $7.736(39)$ & 0.002 & 129.3 & & & \\
\hline $20 / 21$ & SHOC & 1 & 6742 & 1.9 & $6.782(1)$ & 0.008 & 147.4 & & & & & & \\
\hline $21 / 22$ & SHOC & 0.5 & 4115 & 0.6 & $6.791(23)$ & 0.008 & 147.3 & & & & & & \\
\hline May 01/02 & SHOC & 1 & 3600 & 1.0 & $6.786(51)$ & 0.004 & 147.7 & & & & & & \\
\hline 2018 Feb $15 / 16$ & STE3 & 15 & 307 & 1.8 & $6.766(8)$ & 0.005 & 147.8 & $7.530(27)$ & 0.001 & 132.8 & & & \\
\hline $16 / 17$ & STE3 & 15 & 239 & 1.4 & $6.777(10)$ & 0.005 & 147.6 & & & & & & \\
\hline \multicolumn{14}{|l|}{ EC 11545-1459 } \\
\hline 2017 May $17 / 18$ & STE3 & 20 & 297 & 2.2 & $7.227(21)$ & 0.003 & 138.4 & $6.528(16)$ & 0.004 & 153.2 & & & \\
\hline $18 / 19$ & STE3 & 15 & 645 & 3.8 & $7.214(8)$ & 0.004 & 138.6 & $6.521(14)$ & 0.003 & 153.4 & & & \\
\hline $19 / 20$ & STE3 & 20 & 575 & 4.2 & $7.209(5)$ & 0.003 & 138.7 & & $<0.001$ & & & & \\
\hline $20 / 21$ & STE3 & 20 & 337 & 2.5 & $7.240(15)$ & 0.003 & 138.1 & $6.528(12)$ & 0.004 & 153.2 & & & \\
\hline $30 / 31$ & SHOC & 3 & 1200 & 1.0 & & & & $6.565(23)$ & 0.008 & 152.3 & & & \\
\hline 2018 Feb 18/19 & STE3 & 15 & 188 & 1.2 & & & & $6.530(17)$ & 0.011 & 153.2 & & & \\
\hline $19 / 20$ & STE3 & 15 & 183 & 1.3 & & & & $6.514(35)$ & 0.005 & 153.5 & & & \\
\hline Mar 18/19 & STE3 & 15 & 256 & 1.3 & & & & $6.510(16)$ & 0.005 & 153.6 & & & \\
\hline \multicolumn{14}{|l|}{ EC 21281-5010 } \\
\hline 2017 May 20/21 & STE3 & 15 & 158 & 0.9 & $7.159(16)$ & 0.012 & 139.7 & $7.783(14)$ & 0.010 & 128.5 & & & \\
\hline $21 / 22$ & STE3 & 12 & 447 & 2.5 & $7.112(4)$ & 0.013 & 140.6 & $7.762(4)$ & 0.010 & 128.8 & & & \\
\hline $22 / 23$ & STE3 & 12 & 732 & 3.8 & $7.110(2)$ & 0.011 & 140.6 & $7.766(2)$ & 0.010 & 128.8 & & & \\
\hline Oct $10 / 11$ & STE3 & 12 & 808 & 4.1 & $7.112(2)$ & 0.012 & 140.6 & $7.764(2)$ & 0.008 & 128.8 & & & \\
\hline $11 / 12$ & STE3 & 12 & 604 & 3.2 & $7.111(3)$ & 0.011 & 140.6 & $7.763(3)$ & 0.008 & 128.8 & & & \\
\hline
\end{tabular}

We obtained three further runs on the star with SHOC in 2017 January. The first two were on the same night, separated by only a couple of minutes, and the third run was two nights later. In addition we were able to get three runs with the STE3 instrument in 2017 October and three more in 2018 February. Table 2 lists brief observing notes and the extracted frequencies and amplitudes; sample periodograms are shown in Fig. 1, selected to illustrate the range of periodogram variability, and the $2018 \mathrm{Feb}$ periodograms are shown in Fig. 2. The two frequencies found by Kilkenny et al. (2017) are recovered in the two SHOC runs from 2017 Jan 14/15 
mag

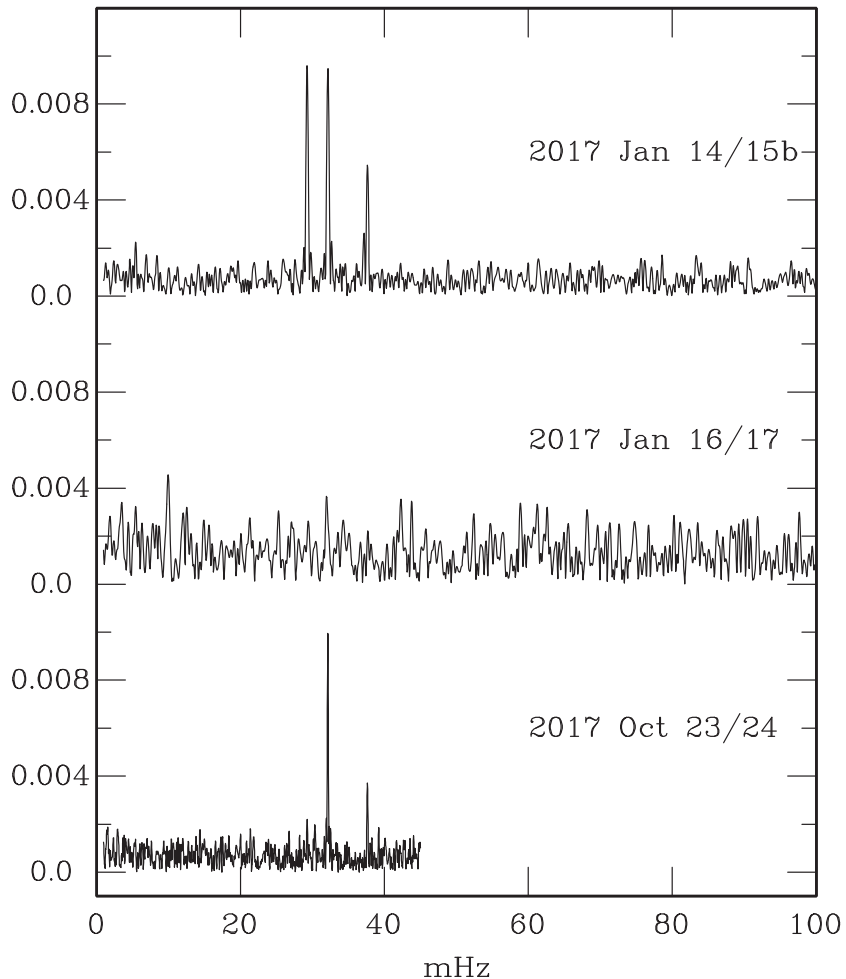

Figure 1. Sample periodograms for EC 03089-6421 from three of the 2017 runs listed in Table 2. The upper two periodograms are from SHOC observations, the lower is from STE3 and is truncated at the Nyquist frequency for those data (equivalent to $22 \mathrm{~s}$ ).

together with a third frequency at $37.6 \mathrm{mHz}$ equivalent to a period of $26.6 \mathrm{~s}$. In the third SHOC run (Fig. 1; middle panel), there is no obvious variation in the light curve and the periodogram shows little of significance. With foreknowledge of the frequencies, it is just possible to see the $32.1 \mathrm{mHz}$ variation in the periodogram; without that knowledge, the star would probably be considered non-variable on the evidence of this periodogram. The 2017 STE3 runs confirm the $37.6 \mathrm{mHz}$ variation but the $34.2 \mathrm{mHz}$ variation is absent or very weak - again, there are peaks in two nights (Oct 10/11, and 23/24) but at only about twice the rms noise level (see the lower panel in Fig. 1) so that these would not be considered real in the absence of other information. The 2018 Feb periodograms (Fig. 2) show the original frequencies as much stronger than previously seen; the 31.1 $\mathrm{mHz}(31.1 \mathrm{~s})$ variation starts at an amplitude of nearly $0.02 \mathrm{mag}$ but within 4 days has fallen to $0.005 \mathrm{mag}$; the $29.2 \mathrm{mHz}(34.2 \mathrm{~s})$ variation starts below $0.02 \mathrm{mag}$ but grows to $0.025 \mathrm{mag}$; the new $37.6 \mathrm{mHz}(26.6 \mathrm{~s})$ variation is almost undetectable at first but grows to an easily visible $0.006 \mathrm{mag}$.

The amplitude changes between the various nights are consistent with and on comparable time-scales to those seen in the discovery paper.

\section{NEW PULSATING B SUBDWARFS}

Table 2 gives some details from the observing logs of the new variables as well as the frequencies extracted on a nightly basis. Fig. 3 shows illustrative light curves for the five $\mathrm{sdBV}_{r}$ stars. Additional comments are listed in the subsections below.

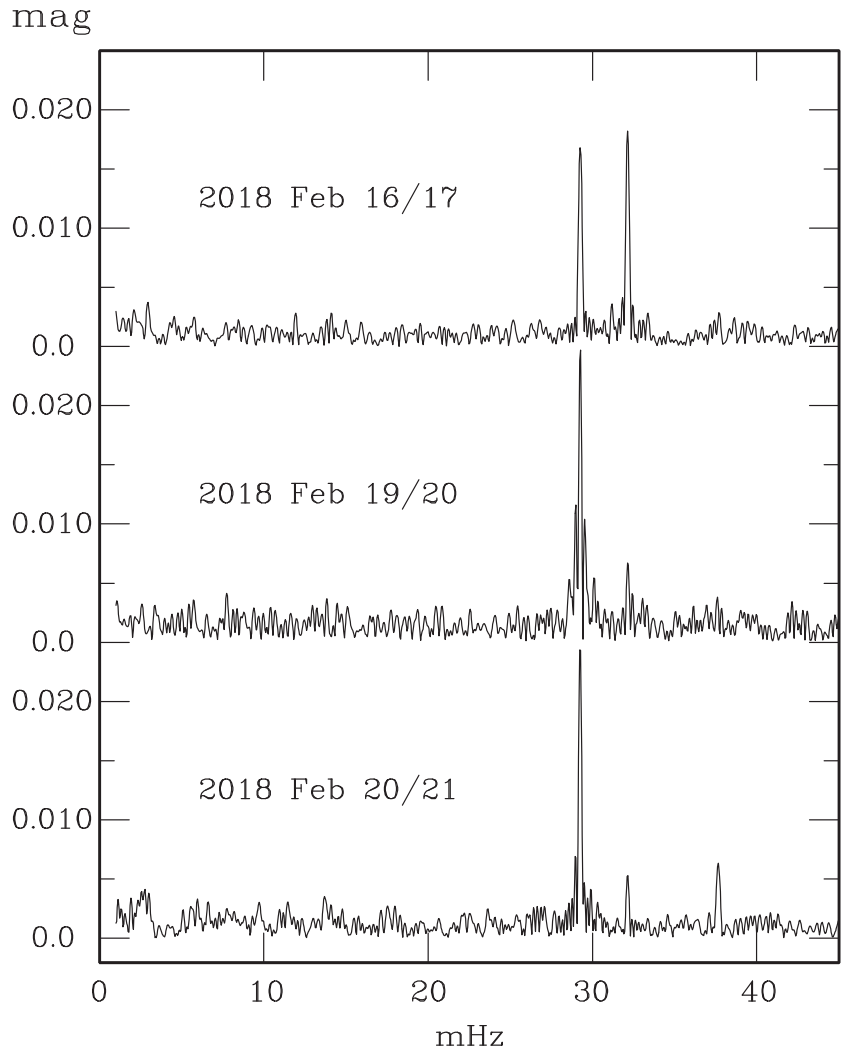

Figure 2. Periodograms for EC 03089-6421 from the 2018 runs listed in Table 2. Note the difference in abscissa scale from Fig. 1.

\subsection{EC 01441-6605}

EC 01441-6605 was the last star discussed in this paper to be identified as a pulsator, and there are only data from one week. All periodograms show three low-amplitude $(\sim 0.005 \mathrm{mag})$ variations near $6.862,7.040$, and $8.125 \mathrm{mHz}(147,142$, and $123 \mathrm{~s}$ periods). $\mathrm{Pe}-$ riodograms from the three longest runs are shown in Fig. 4 and, from the limited baseline of our observations, the amplitudes appear constant. If this is verified, the star would be a suitable target for continued occasional monitoring. EC 01441-6605 was classified sdB + F in the EC survey (Kilkenny et al. 2016) and thus might exhibit the same sort of pulsation phase changes - due to the binary orbit - as recently demonstrated for EC 20117-4014 (Otani et al. 2018).

The periodogram derived from combined data from the four consecutive nights yields $6.8660 \pm 0.0001 \mathrm{mHz}(145.65 \mathrm{~s}$; $0.0036 \pm 0.0002 \mathrm{mag}), 7.0385 \pm 0.0001 \mathrm{mHz}(142.08 \mathrm{~s}$; $0.0047 \pm 0.0002 \mathrm{mag}$ ), and $8.1215 \pm 0.0002 \mathrm{mHz}(123.13 \mathrm{~s}$; $0.0023 \pm 0.0002 \mathrm{mag}$ ), though with data trains which are short compared to the night-to-night gaps, there is always the danger of aliasing at multiples of $0.01157 \mathrm{mHz}$.

\subsection{EC 10384-1301}

EC 10384-1301 has only a single comparison star on the STE3 frames; this is significantly fainter and so introduces unacceptable noise if used to correct the data. Uncorrected data from a single short STE3 run on an apparently photometric night in late May shows only a single frequency at $6.940 \pm 0.017 \mathrm{mHz}($ period $=144.1 \mathrm{~s})$ with a substantial amplitude of $0.011 \mathrm{mag}$ (see the upper panel of Fig. 5). Two SHOC runs shortly afterwards appeared to confirm this, although there was weak evidence for other significant peaks. 


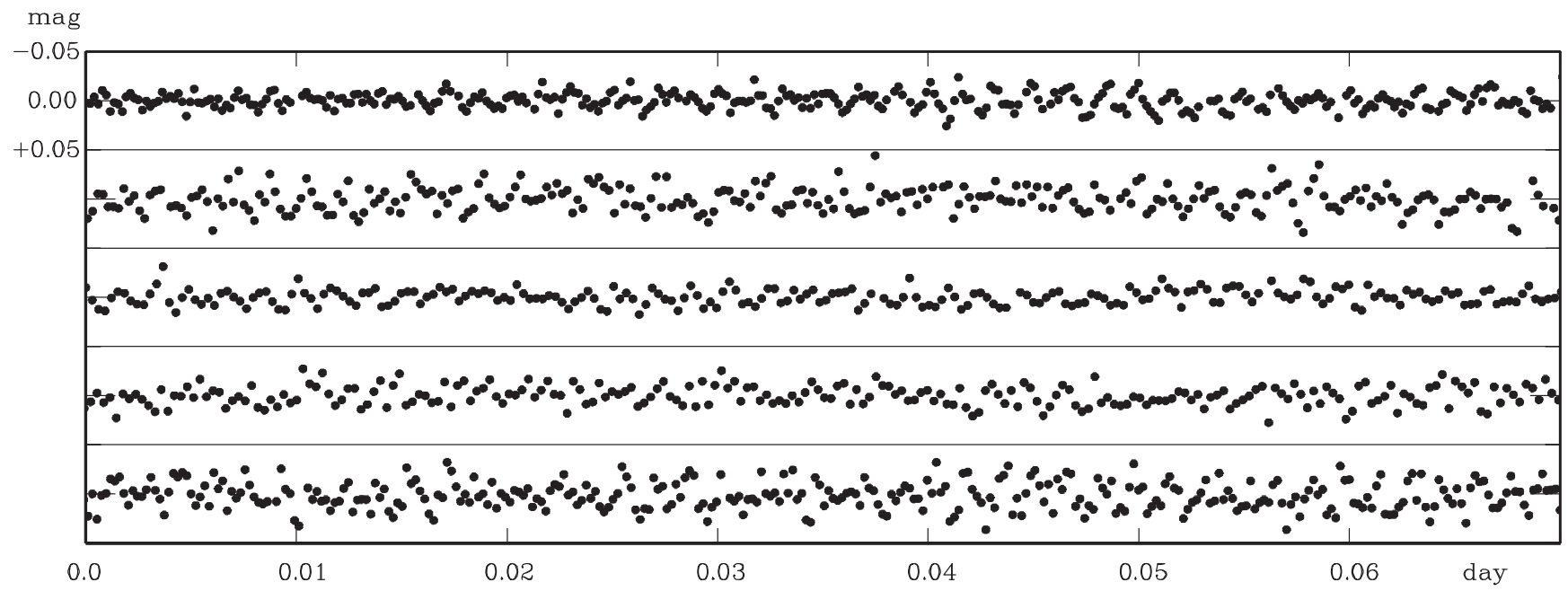

Figure 3. Illustrative sections of STE3 light curves for the new $\mathrm{sdBV}_{r}$ variables. From top to bottom, the stars are EC 01441-6605, 10384-1301, 11275-2504, 11545-1459, and 21281-5010. Abscissae are $\sim 1.7 \mathrm{hr}$ in length and the observations have had the mean magnitude for each light curve removed. The ordinate scale is the same for each panel.

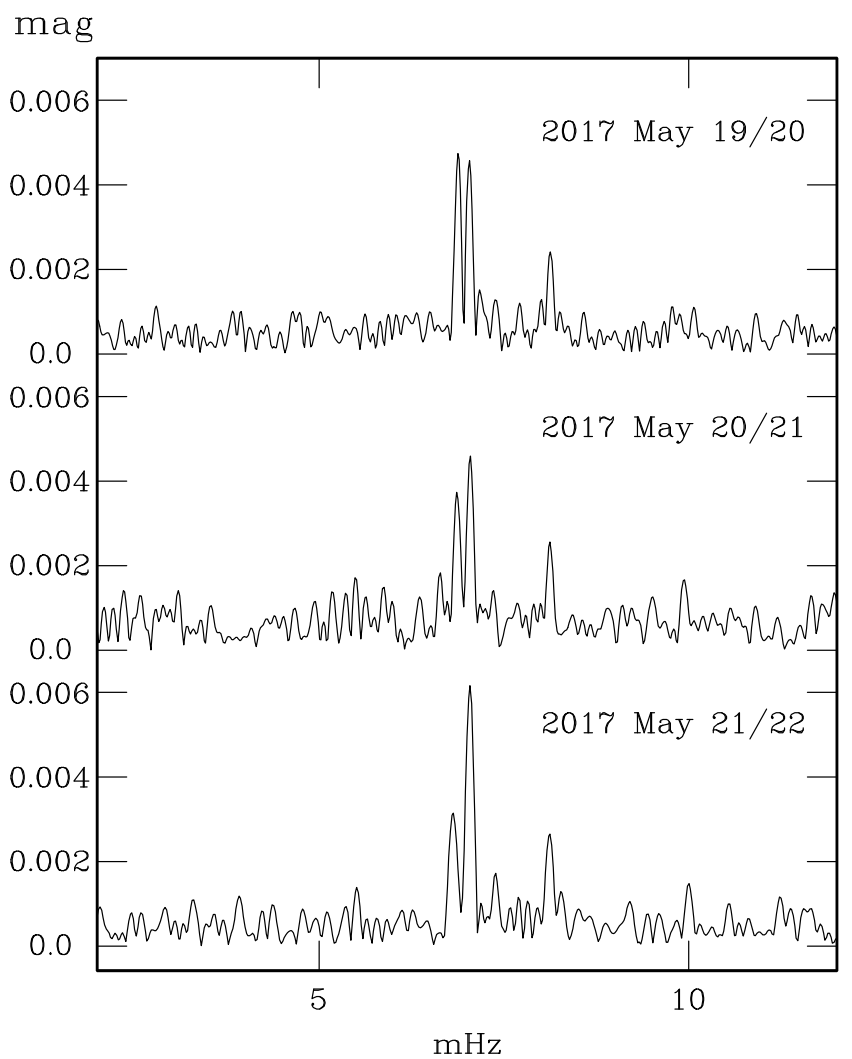

Figure 4. Sample periodograms for EC 01441-6605 from three of the runs listed in Table 2. The frequency scale is expanded relative to other figures, better to separate the two close frequencies near $7.0 \mathrm{mHz}$.

Two further STE3 runs in 2018 Feb show different results (lower panel in Fig. 5). The previously seen strong peak has fallen to half its 2017 amplitude and two weaker peaks $(\sim 0.004$ and $0.008 \mathrm{mag}$ ) appear near 6.57 and $6.70 \mathrm{mHz}$, respectively (152 and 148 s). Comparing 2018 and 2017 runs gives more credibility to the weak peaks. In any case, the star clearly exhibits amplitude variability.

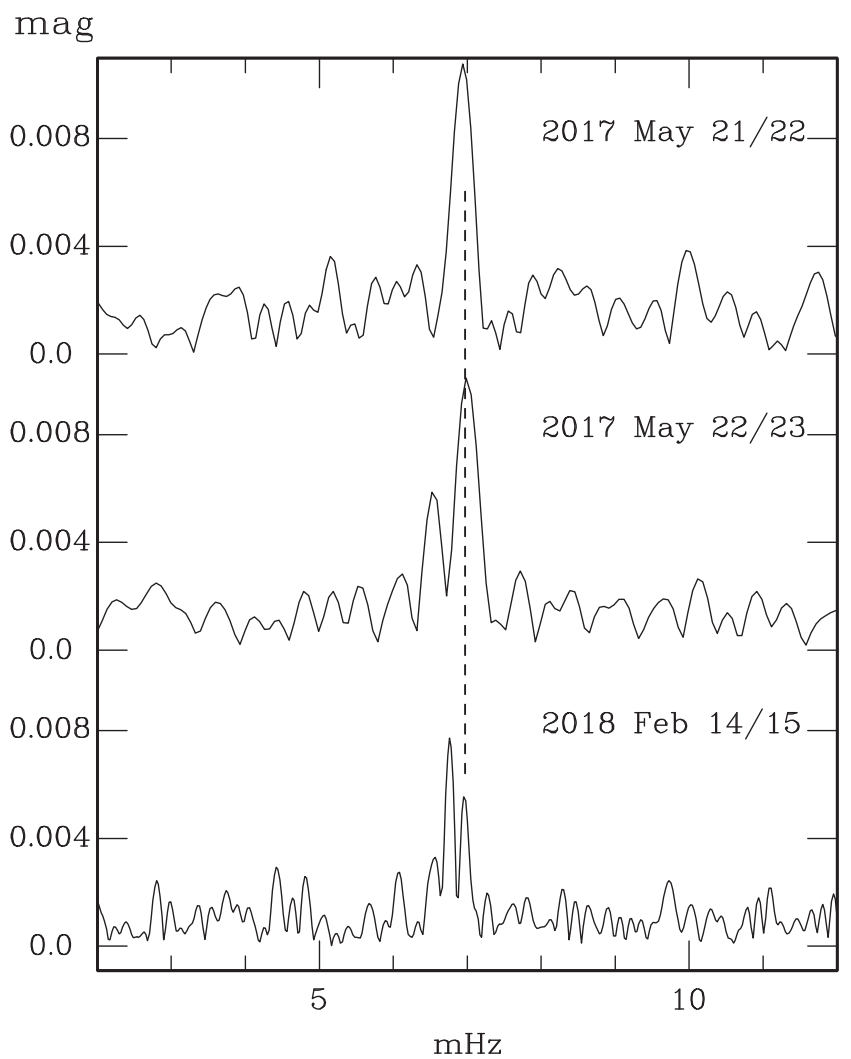

Figure 5. Sample periodograms for EC 10384-1301. The horizontal scale is expanded compared to similar plots for the other stars. The dashed line indicates the variation near $7.0 \mathrm{mHz}(\sim 143 \mathrm{~s})$.

\subsection{EC 11275-2504}

EC 11275-2504 was the last target observed on the last night of an STE3 run in 2016 February and was discovered to be variable, but it was not possible to observe the star again until early 2017 when it was observed on four nights using SHOC. Two more runs were obtained in 2018 February. Sample periodograms are shown in Fig. 6 and the derived frequencies listed in Table 2. There is a 
mag

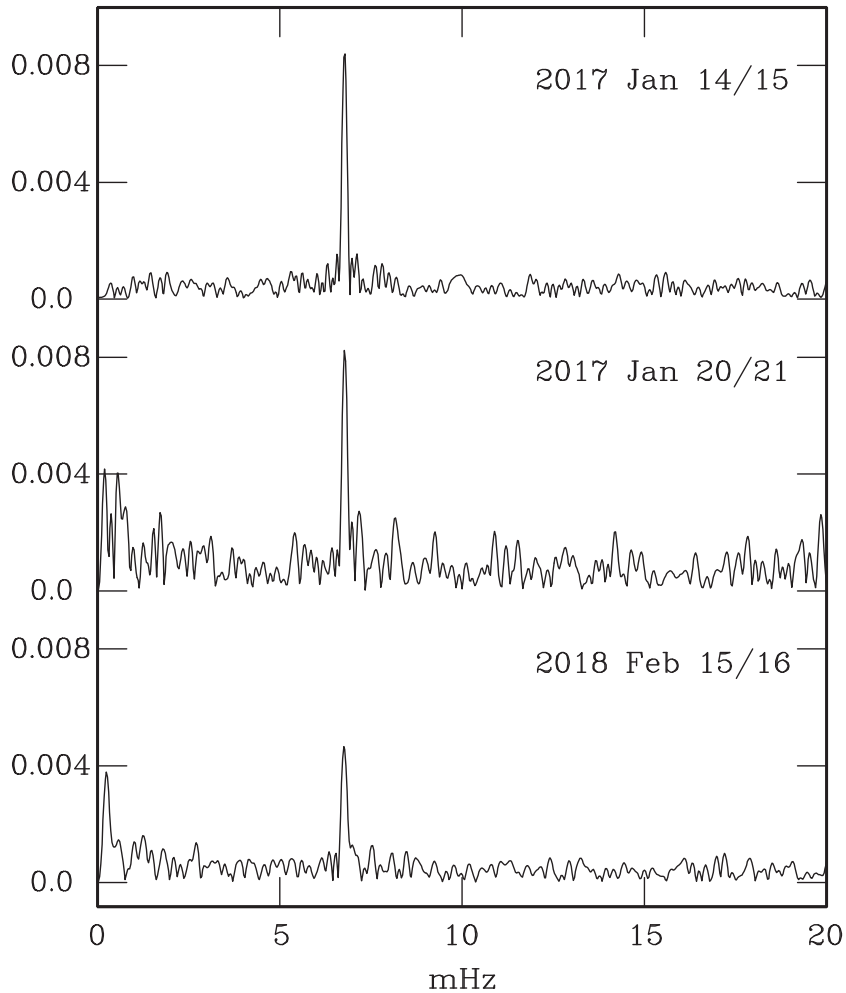

Figure 6. Sample periodograms for EC 11275-2504 from STE3 (upper) and SHOC (middle and lower).

clear single frequency near $6.780 \mathrm{mHz}(147.5 \mathrm{~s})$ with an amplitude of $0.009 \mathrm{mag}$ in 2017 , dropping to $0.005 \mathrm{mag}$ a year later. In four of the periodograms there is a very weak signal near 7.70 $\mathrm{mHz}(130 \mathrm{~s})$, but the frequency differences from run to run are large compared to the formal errors of the frequency determination (Table 2) and the amplitudes are only 0.001-0.002 mag which is only about twice the noise (see Fig. 6). Based on the current data therefore we consider EC 11275-2504 to be singly periodic, although the possible existence of a second period is entertained again in Section 7.

\subsection{EC 11545-1459}

EC 11545-1459 has two possible comparison stars on STE3 frames; one is too bright and saturates at useful exposures and the other clearly variable in all our runs - is a known RR Lyrae variable from the Catalina Real-Time Survey (CRTS J115656.4-151648; Drake et al. 2009). The lack of suitable comparisons is unfortunate, as the star is the faintest on our list - so the noise in the data is more significant - and the derived variations are of small amplitude, but we have had to use uncorrected data from photometric nights for the following analysis

Seven STE3 runs and a SHOC run on EC 11545-1459 are listed in Table 2, five from 2017 May, and three from early 2018, and a sample of periodograms is shown in Fig. 7. Two small-amplitude variations are detected near $7.22 \mathrm{mHz}(138.5 \mathrm{~s} ; 0.003 \mathrm{mag})$ and 6.53 $\mathrm{mHz}$ (153.1 s; $0.003 \mathrm{mag}$ ) although on May 19/20, the latter is too weak to be detected at about the $0.001 \mathrm{mag}$ level (see the middle panel in Fig. 7) and in the 2018 data, the former is not detected at all. Variations with amplitudes $\sim 0.003$ or 0.004 mag might be regarded as uncertain detections - in the case of EC 11545-1459, this is only

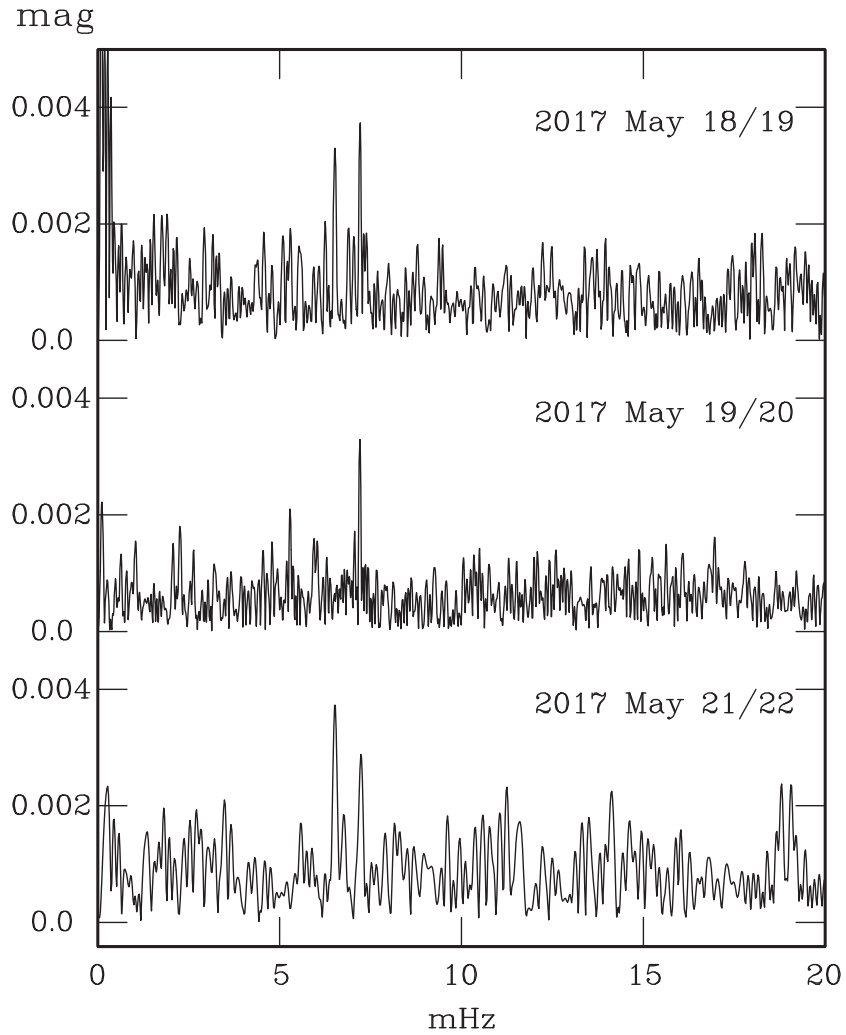

Figure 7. Sample periodograms for EC 11545-1459, including that for the May $19 / 20$ data, where the variation near $6.53 \mathrm{mHz}(153.3 \mathrm{~s})$ is too small to be detected above noise.

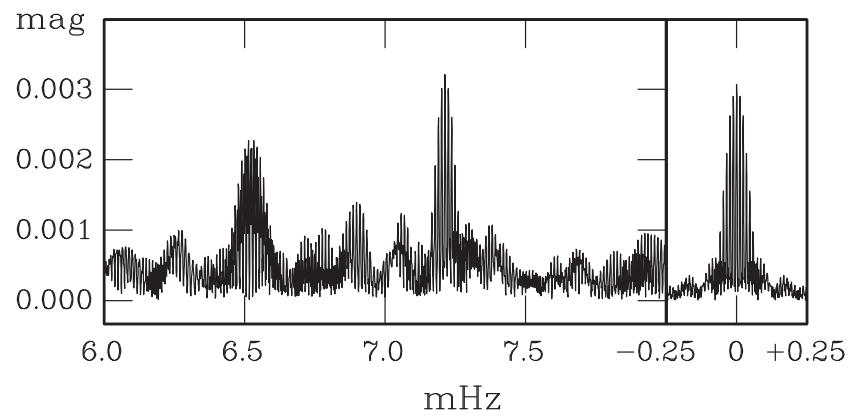

Figure 8. Periodogram for EC 11545-1459 for the four successive STE3 nights in 2017 May (17/18-20/21) shown on a much expanded scale to Fig. 7.

about 3 or 4 times the background noise in the periodograms (Fig. 7) - but for the fact that these same frequencies are found (within the uncertainties) in most of the periodograms.

As noted at the end of Section 1, individual observing runs are generally too short and separated to give any additional information when combined (except perhaps for increasing the accuracy of the frequency determination - subject to the problem of aliasing). EC 11545-1459 has perhaps the best chance of doing this, having four somewhat longer STE3 runs on consecutive nights in 2017. The periodogram for these combined is shown in Fig. 8 and yields: $7.2131 \pm 0.0002 \mathrm{mHz}(138.6 \mathrm{~s} ; 0.0032 \pm 0.0004$ $\mathrm{mag}$ ); the frequency near $6.53 \mathrm{mHz}$ splits into $6.5327 \pm 0.0003$ $\mathrm{mHz}(153.0 \mathrm{~s} ; 0.0023 \pm 0.0004 \mathrm{mag}) ; 6.5252 \pm 0.0004 \mathrm{mHz}$ (153.2 s; $0.0020 \pm 0.0004)$ reflecting the clear variability seen in the amplitude (Fig. 7); and a weak frequency may be present at 


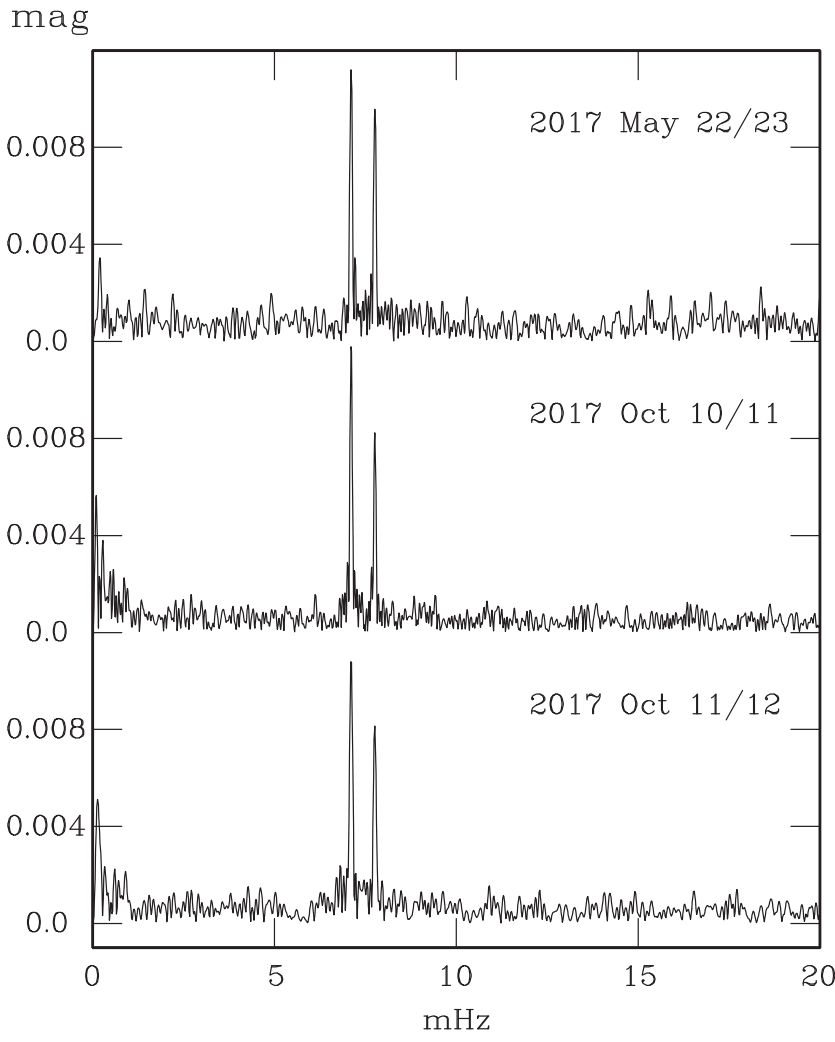

Figure 9. Sample periodograms for EC 21281-5010 from the longest three runs in Table 2.

$6.8982 \pm 0.0006 \mathrm{mHz}(145.0 \mathrm{~s} ; 0.0013 \pm 0.0004)$ though this is hardly three times the background noise and thus suspect.

\subsection{EC 21281-5010}

Even on the STE3 camera, EC 21281-5010 has four comparison stars of comparable brightness and so differentially corrected data were used, allowing analysis of two poor nights as well as a photometric one. Two strong frequencies are detected near 7.11 $\mathrm{mHz}$ (140.6 s, $0.012 \mathrm{mag})$ and $7.769 \mathrm{mHz}(128.7 \mathrm{~s}, 0.010 \mathrm{mag})$. A weak frequency was detected near $7.398 \mathrm{mHz}(135.2 \mathrm{~s}, 0.003$ mag), but only on one night, so is unlikely to be real. On the three consecutive nights of observation in 2017 May and two nights in 2017 October, there is no strong indication of amplitude variability (see Fig. 9).

From the three STE3 runs on consecutive nights, we have calculated the periodogram for the combined data and find frequencies of $7.1102 \pm 0.0001 \mathrm{mHz}(140.6 \mathrm{~s}, 0.0117 \pm 0.0004 \mathrm{mag})$ and $7.7688 \pm 0.0001 \mathrm{mHz}(128.7 \mathrm{~s}, 0.0099 \pm 0.0004 \mathrm{mag})$ in good agreement with the two longest runs (Table 2).

\section{THE BINARY SDB, EC 15061-1442}

EC 15061-1442 (= LB 283) was detected as variable nearly 10 years ago but, as we have made little progress since the discovery, we publish the simple fact here. The STE3 field contains one star bright enough to be used as a comparison star but correction by this star seems to result in the introduction of slow drifts into the data so we have used uncorrected data. Two similar runs on successive nights in 2008 Jun (Fig. 10) indicate a period of $\sim 0.075 \mathrm{~d}$ if the star is a reflection effect binary and $0.15 \mathrm{~d}$ if an ellipsoidal variable. mag

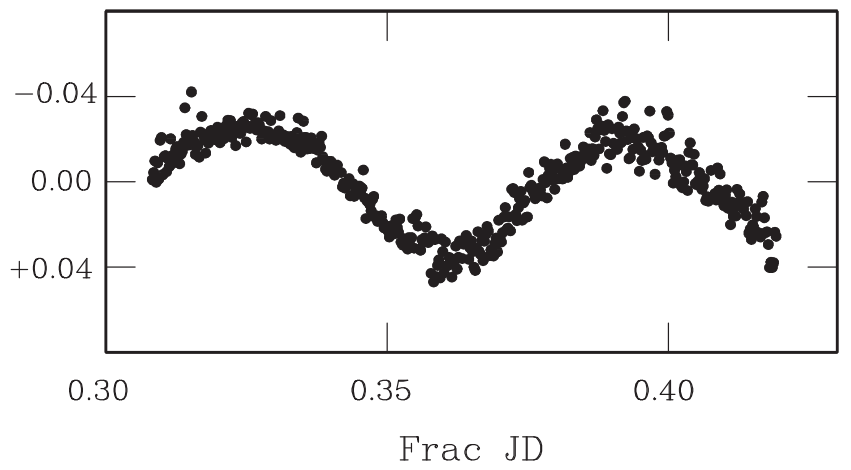

Figure 10. Light curve for EC 15061-1442 from 2008 Jun 29/30.

If the former, the binary is at the very low end of known orbital periods (Heber, 2016).

\section{SPECTROSCOPY}

Energy distributions for our targets were assembled using photometry taken from a variety of sources. The revised catalogue of GALEX Ultraviolet Sources (Bianchi, Shiao \& Thilker 2017) provided monochromatic ultraviolet fluxes at effective wavelengths of 1528 and 2310 Å for each object, except EC 11275-2504 which was judged to be too close to the detector edge to provide useful results. Near-infrared fluxes were obtained at effective wavelengths of $1.25,1.65$ and $2.16 \mu \mathrm{m}$ using the Cohen, Wheaton \& Megeath (2003) calibration of the Two Micron All Sky Survey (Skrutskie et al. 2006; 2MASS) photometry. Wright et al. (2010) describe the WideField Infrared Survey Explorer (WISE) and its flux calibration; this gives far-infrared fluxes at 3.4 and $4.6 \mu \mathrm{m}$ for stars listed in Table 1 . Finally, fluxes at effective wavelengths of 3660,4380 and $5450 \AA$ were obtained for each object, using Table 1 UBV photometry and the Bessell, Castelli \& Plez (1998) calibration.

Stellar effective temperature $\left(T_{\text {eff }}\right)$ determinations based on photometry or spectral energy distributions are dependent on knowing interstellar reddening, $E(B-V)$, along the line of sight. A distance was obtained for each star from the GAIA-distance catalogue by Bailer-Jones et al. (2018) and used to obtain $E(B-V)$ with the Galactic extinction model by Amôres and Lépine (2005).

EC survey low-dispersion $\left(\sim 100 \AA \mathrm{mm}^{-1}\right)$ classification spectra (Stobie et al. 1997) of EC 01441-6605 (O'Donoghue et al. 2013); EC 10384-1301, EC 11275-2504, EC 11545-1459, and EC 150611442 (Kilkenny et al. 1997b) were used to determine effective temperatures $\left(T_{\text {eff }}\right)$, surface gravities $(\log g)$, and helium number fractions $N(\mathrm{He}) / \mathrm{N}(\mathrm{H})$ for the photospheres of these stars. The EC survey classification spectrum of EC 21281-5010 (Kilkenny et al. 2015) was no longer available and its $T_{\text {eff }}$ was determined by Remie and Lamers (1982) iteration. EC 03089-6421 is known (Kilkenny et al. 2017) to be an sdO pulsator and is therefore a special case discussed below.

As EC survey spectra were not necessarily obtained in photometric conditions, available photometric flux densities detailed above were represented by a seventh-order polynomial obtained by least squares; this was divided by a manually fitted continuum, based on the Hill (1982) routine INTEP, giving a wavelengthdependent function by which the corresponding observed spectrum was multiplied. The adopted procedure corrects also for wavelengthdependent light losses at the spectrograph slit. Applying a flux density $\left(F_{\lambda}\right)$ shift to the resulting spectrum, ensuring agreement 
with the dereddened Johnson-B filter $F_{4380}$, corrects for a systematic error arising from use of the continuum.

Latour et al. (2011) demonstrate the atmospheric structure dependence on line-blanketing when model stellar atmospheres are computed without the assumption of local thermodynamic equilibrium (non-LTE). We have nonetheless taken our model nonLTE spectra from the hydrogen-helium grid by Németh et al. (2014), as there were no metal lines attributable to hot subdwarfs in our spectra distinguishable from noise. (An exception is EC 030896421 which is discussed below). As Latour et al. (2011) show, the neglect of metals is primarily seen in line cores and so in making our fits, we made no attempt to fit line cores but instead focused on securing good fits to line wings. EC 01441-6605 and EC 150611442 are binaries and for these cases, PHOENIX specific intensity $1 \AA$ resolution spectra by Husser et al. (2013) were integrated over all 78 angle points to provide model cool companion solar metallicity spectra.

The neglect of Balmer line cores in particular, required that all fits were made by hand. Model spectra for a range of trial $T_{\text {eff }}, \log g$, and $N(\mathrm{He}) / \mathrm{N}(\mathrm{H})$ values were tried after scaling by the square of the implied angular radius $(\alpha)$. Adopted fits are shown in Fig. 11 with scaling parameters given in Table 3 .

In Fig. 11 , it can be seen that Ca II K $(\lambda 3933 \AA)$ is present in the spectrum of EC 11545-1459, which of course could not be fitted as the model included only a single sdB star. The strength of the CaII K feature suggested, as in the case of EC 014416605, an F-type main sequence companion but photometry by Kilkenny et al. (1997b), reproduced in Table 1, is inconsistent with this possibility. As those authors necessarily made photometric and spectroscopic observations of their targets at single epochs, EC 11545-1459 could be a binary whose cool companion was eclipsed at the photometric epoch but not at the spectroscopic epoch.

As a check on the possibility of an eclipse at the time the EC survey photometry was obtained, we took GAIA photometry of EC 11545-1459 and BD + 17 4708 from Evans et al. (2018). $\mathrm{BD}+17^{\circ} 4708$ is a fundamental photometric standard for which Bohlin and Gilliland (2004) publish an energy distribution. Weiler (2018) gives bandpasses for the GAIA photometric filters from which a GAIA flux calibration (effective filter wavelengths and zeropoints) was derived using the Bohlin and Gilliland (2004) energy distribution for BD $+17^{\circ} 4708$. As sdB stars have a higher $T_{\text {eff }}$ than $\mathrm{BD}+17^{\circ} 4708$, a small colour correction would be needed when applying the derived GAIA flux calibration to these stars; adopting this to be zero showed that GAIA and EC survey photometry for EC 11545-1459 were consistent. It was therefore considered unlikely that the sdB EC 11545-1459 was eclipsed by a late-type companion at the time EC survey photometry was obtained and the Ca II $(\mathrm{K})$ line in its spectrum remains unexplained.

The model spectrum for EC 10384-1301 in Fig. 11 was based on the assumption that the He II $\lambda 4686 \AA$ line is real and not a noise feature, which was in doubt given that the original spectrum had a maximum $S / N \sim 12$ at $\mathrm{H} \delta$. Model spectrum Balmer lines and He II $\lambda 4686 \AA$ were weaker than those in the observed spectrum. Lowering $T_{\text {eff }}$ would weaken He II $\lambda 4686 \AA$ and make He I lines too strong. Raising $T_{\text {eff }}$ would make He II $\lambda 4541 \AA$ stronger than observed, while weakening the Balmer lines further. There was similarly little scope for adjusting the helium abundance and the adopted model spectrum was a compromise. In the absence of further photometric and spectroscopic observations, claiming EC 10384-1301 as a new sdO pulsator could not be justified.
Kilkenny et al. (2017) identify EC 03089-6421 as a rare example of a rapidly pulsating field sdO star (Heber 2016); this clearly distinguishes it from the other stars discussed in the present paper. While Kilkenny et al. (2017) obtain three higher resolution spectra, in addition to the EC survey spectrum already available, their analysis leads to discordant $T_{\text {eff }}$ values (see their fig. 8). Fitting He II $\lambda 4686 \AA$ gives $T_{\text {eff }}=85000 \mathrm{~K}$ whereas fitting Balmer lines gives $T_{\text {eff }}=38000 \mathrm{~K}$; in the latter case, He II $\lambda 4686 \AA$ is weaker than observed and He I $\lambda 4472 \AA$ much stronger. Metal line blanketing is not taken into account in the Stroeer et al. (2007) nonLTE grid, which Kilkenny et al. (2017) use and therefore (as argued above) fitting line-cores, rather than just line-wings, leads them to the difficulty they show in their fig. 8 .

We found no significant radial velocity shifts between the three Grating 4 spectra obtained by Kilkenny et al. (2017), and so we added these without applying relative velocity shifts and used the result for comparison with model spectra. A flux calibration was applied, after correcting for interstellar reddening, using the procedure described above. Based on the grid of non-LTE HHe synthetic spectra by Németh et al. (2014) and not having concerned ourselves with fitting line-cores, we obtained the fit for $T_{\text {eff }}=52000 \mathrm{~K}$ presented in Fig. 12 .

For EC 03089-6421, $T_{\text {eff }}$ is defined by the need to fit He II $\lambda 4686$ $\AA$ and having $T_{\text {eff }}$ high enough and low enough, respectively, to ensure that neither He I $\lambda 4472 \AA$ nor He II $\lambda 4541 \AA$ are too strong in the model spectrum. The helium abundance was similarly constrained. Even so, the adopted fit was a compromise because the model spectrum He II $\lambda 4686 \AA$ line is too weak and both He I $\lambda 4472$ $\AA$ and He II $\lambda 4541 \AA$ are stronger than observed. Model Balmer lines were also too weak; only the wings of $\mathrm{H} \delta, \mathrm{H} \gamma$ and the blue wing of $\mathrm{H} \beta$ have been fitted.

Confirmation of the need to include metal-line blanketing in nonLTE model stellar atmospheres for EC 03089-6421, comes from the feature near $\lambda \sim 4632 \AA$ which appeared to be real as it was prominently visible in all four available spectra. Latour et al. (2014) attribute to N III a line at a similar wavelength in their spectrum of the star 177238 in $\omega$ Cen, which has atmospheric parameters similar to those we found for EC 03089-6421. We have therefore tentatively identified the feature near $\lambda \sim 4632 \AA$ as being partly due to N III but ultraviolet spectra are needed to identify other metal contributions; this would be an essential prerequisite to calculating a more realistic model spectrum for EC 03089-6421 based on a line-blanketed non-LTE model atmosphere.

As the EC survey spectrum of EC 21281-5010 is no longer available, its $T_{\text {eff }}$ had to be determined using photometry alone. The EC-type and UBV photometry which Kilkenny et al. (2015) obtain, as quoted in Table 1, suggested a similarity between EC 212815010 and EC 11275-2504, which would imply similar atmospheric parameters. The Remie and Lamers (1982) iteration gave $T_{\text {eff }} \simeq$ $35000 \mathrm{~K}$ for $E(B-V)=0.03$, but comparisons with spectroscopic determinations of $T_{\text {eff }}$ for other apparently single stars studied in this paper suggested that the Remie and Lamers iteration (1982) did not yield high-quality determinations, at an assumed $E(B-$ $V$ ), when $G A L E X$ photometry was used instead of international ultraviolet explorer low-resolution spectra. Moreover, $T_{\text {eff }}$ and $E(B$ $-V)$ are highly correlated when the former was determined using the Remie and Lamers (1982) iteration and an uncertain $E(B-V)$ translated directly into an uncertain $T_{\text {eff }}$.

Derived atmospheric parameters are listed in Table 4. Atmospheric parameter errors were estimated in each case by perturbation; each parameter was varied in turn, holding the others fixed, until the associated fit in Figs 11 or 12 was noticeably degraded. 




Figure 11. The comparison between dereddened observed (red) and model (cyan) spectra. Values for the scaling parameters $\beta$ and $\gamma$ are presented in Table 3 . Note that observed spectra have a resolution of $\sigma=0.85 \AA$ and have been further convolved with a Gaussian having $\sigma=2 \AA$ to enhance signal to noise ( $\mathrm{S} / \mathrm{N}$ ). Model spectra have been smoothed to match. Further details are given in the text.

Table 3. Scaling parameters used in Fig. 11. $\beta$ is dimensionless and $\gamma$ is expressed in ergs $\mathrm{cm}^{-2} \sec ^{-1} \AA^{2}$.

\begin{tabular}{lcc}
\hline EC & $\gamma$ & $\beta$ \\
\hline $01441-6605$ & -0.6 & $1.429 \times 10^{3}$ \\
$10384-1301$ & +0.1 & $2.326 \times 10^{3}$ \\
$11275-2504$ & +0.7 & $4.545 \times 10^{2}$ \\
$11545-1459$ & +1.3 & $3.030 \times 10^{3}$ \\
$15061-1442$ & +2.0 & $2.778 \times 10^{2}$ \\
\hline
\end{tabular}

As $\alpha$ and $T_{\text {eff }}$ are highly correlated, errors in $\alpha$ are likely to be underestimated as they were determined at a fixed $T_{\text {eff }}$.

\section{DISCUSSION}

Distances for hot subdwarfs, found to pulsate through work reported in the present paper, were obtained from the compilation by BailerJones et al. (2018) and are presented in Table 5. Quoted error limits were obtained as half the differences between the maximum and minimum distances Bailer-Jones et al. (2018) report. Hot subdwarf radii $(R)$, masses $(M)$, and luminosities $\left(\log _{10} L\right)$ then followed from spectroscopically derived parameters presented in Table 4 and are included in Table 5. An exception was EC 212815010 for which we had no $\log g$ determination and therefore no mass.

The special case of EC 03089-6421 is discussed below; excluding this and EC 10384-1301, for which the mass uncertainty and mass were found to be essentially equal, the mean of the derived masses in Table 5 for the remaining four sdB stars was $\bar{M}=0.33 \pm 0.09 \mathrm{M}_{\odot}$. Fontaine et al. (2012) take sdB star masses from the 15 asteroseismic studies available at the time and find a sharply peaked mass distribution having a mean value of $0.47 \mathrm{M}_{\odot}$ with 68.3 per cent of stars in the range $0.441-0.499 \mathrm{M}_{\odot}$. Our value of $\bar{M}$ is significantly different from the asteroseismic mean sdB mass by Fontaine et al. (2012), which has come to be regarded as a 'canonical mass' for sdB stars.

While systematic errors in our mass determinations almost certainly exist and are discussed in the next section, there are observational and theoretical results suggesting a revision to the canonical sdB star mass may prove necessary. For example, while sdB star evolution paths and positions in the $\log g-T_{\text {eff }}$ diagram by Schindler, Green \& Arnett (2015) are consistent with earlier results; their masses are nearly always lower than the median of the empirical sdB star mass distribution. Another case is the Preece, Tout \& Jeffery (2018) consideration of tidal effects in close binaries of the HW Vir type, in which one component is a sdB star and the other a low-mass MS star, showing that none of the systems they study should be tidally-locked if the $\mathrm{sdB}$ component has the canonical mass of $0.47 \mathrm{M}_{\odot}$. A third example is the Baran et al. (2018) use of the Rømer delay they obtain from a precision Kepler light curve, showing that the $\mathrm{sdB}$ star in the HW Vir system has a mass of $0.26 \mathrm{M}_{\odot}$; this they argue is almost certainly too small although a mass of $0.47 \mathrm{M}_{\odot}$ cannot be easily reconciled with the Rømer delay they observe.

Fundamental parameters for cool companions are presented in Table 6. Uncertain $\log g$ determinations translated directly into highly uncertain masses, although these turned out to be $\sim 1 \mathrm{M}_{\odot}$ as expected for a main sequence $\mathrm{F}, \mathrm{G}$, or $\mathrm{K}$ star. $\mathrm{A}$ Hertzsprung-Russell Diagram summarizing the results is presented in Fig. 13, where the hot subdwarf stars form an extended horizontal-branch - as expected. The two cool companions identified in the present paper are located on or close to the main sequence. 


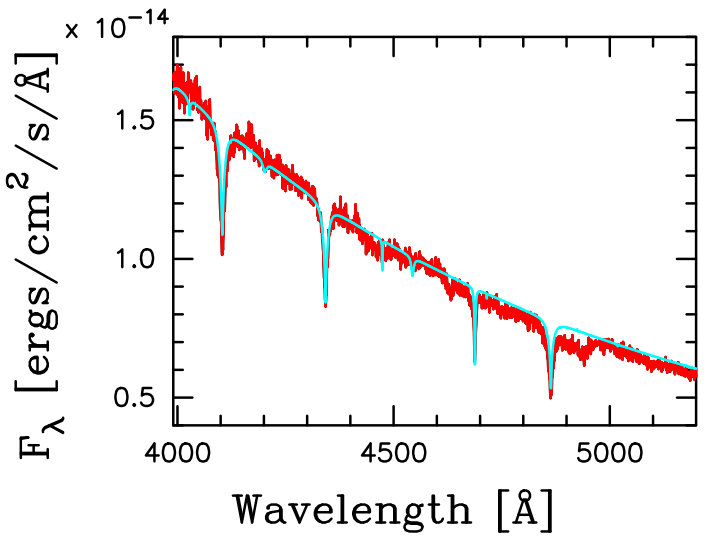

Figure 12. A comparison between the dereddened observed (red) spectrum of EC 03089-6421 and the model (cyan) spectrum. Note that the observed spectrum has a resolution of $\sigma=0.42 \AA$ and the model spectrum has been smoothed to match. No additional smoothing has been applied in this case.

While EC 03089-6421 also lies on the extended horizontal branch in Fig. 13, its low mass suggests that it may be a progenitor to an extremely low-mass white dwarf (ELM WD); if so, it would be expected to reside in a binary system. Kilkenny et al. (2017) obtained their three Grating 4 spectra consecutively on a single night and these showed no significant relative radial velocity shifts; the mean of these, however, is shifted by $-318.6 \mathrm{~km} \mathrm{~s}^{-1}$ with respect to the Grating 6 spectrum obtained by Kilkenny et al. (2015). Trusting the wavelength calibrations, we therefore considered the plausible evolution of EC 03089-6421 in a binary system. A binary companion to the EC 03089-6421 progenitor would have to have removed its envelope at the end of the main sequence evolution in order to produce such a low-mass object. The companion progenitor was presumably a more massive star which had previously evolved into a massive white dwarf, which would not be directly observable on account of its very small radius.

$\mathrm{Li}$ et al. (2019) consider ELM WD formation in doubledegenerate binaries of the type discussed above. The highest mass for EC 03089-6421 allowed by our error bars is $0.16 \mathrm{M}_{\odot}$, which is close to the lowest mass Li et al. (2019) discuss. ELM WDs having $M \leq 0.22 \mathrm{M}_{\odot}$ are found to form through Roche Lobe overflow. Comparing our determinations of $T_{\text {eff }}$ and $\log _{10} \mathrm{~L} / \mathrm{L}_{\odot}$ for $\mathrm{EC}$ 03089-6421 with evolutionary tracks by Li et al. (2019; their fig. 6, upper panel) suggests it is close to its maximum $T_{\text {eff }}$ after a loop of hydrogen-shell flashes.

A further indication of internal structure differences which distinguish EC 03089-6421 from EC 01441-6605, EC 112752504, and EC 11545-1459 came from our plot of periods against a scaled reciprocal square-root of the mean-density $(1 / \tilde{\rho})^{1 / 2}$ shown in Fig. 14; near linear relations would be expected as Eddington $(1918,1919)$ demonstrates. Horizontal error bars are the $(1 / \tilde{\rho})^{1 / 2}$ uncertainties deduced from estimated errors in the masses and radii given in Table 5. Vertical error bars showing period uncertainties are approximately the diameters of the plotted points. Column 8 (black) points for EC 01441-6605, EC 11275-2504, and EC 11545-1459 form a straight line as do those (red) from Column 11; this suggests that the two columns each give periods for a distinct radial overtone in all three stars. Lines representing distinct radial overtones in EC 01441-6605, EC 11275-2504, and EC 11545-1459 do not pass through the EC 03089-6421 points in the lower left-hand corner of Fig. 14, suggesting different pulsation properties in the latter star reflecting an internal structure distinguishing it from hot subdwarfs. Alternatively, Fig. 14 may be regarded as a plot of period against a scaled mean sound-speed characterizing resonant cavities; those for the three hot subdwarfs being clearly distinguished from the one for EC 03089-6421.

EC 15061-1442 appears to be a reflection effect or ellipsoidal binary for which white-light photometry was obtained on Julian Dates 2454646 and 2454647 as discussed above. Dworetsky's (1983) string length method gives an orbital period of $0.0706 \pm 0.0001 \mathrm{~d}$ for the reflection effect case. No attempt was made to analyse the EC 15061-1442 binary as multiband photometry and radial velocity data have yet to be obtained. But calculating a theoretical phased white-light curve assuming it to be a Johnson-B Band light curve, for the reflection effect case, provides insight into the nature of the EC 15061-1442 system. Phased observed and theoretical light curves are compared in Fig. 15.

The 2010 version of the Wilson-Devinney code (Wilson \& Devinney 1971; Wilson 1990, 2008) was used with hot subdwarf parameters from Table 5. In order to match the amplitude of the reflection effect, it was clear that the cool companion has a smaller $\left(\simeq 0.16 \mathrm{M}_{\odot}\right)$ mass and fills its Roche lobe; its $T_{\text {eff }}=5500 \mathrm{~K}$ was adopted from Table 6 . The two stars were found to be separated by $\simeq 0.6 \mathrm{R} \odot$ and have an orbital plane almost orthogonal to the line of sight $\left(\sin i \simeq 15^{\circ}\right)$.

According to our analysis, EC 15061-1442 is an extreme HW Virtype binary in which the hot subwarf is accreting mass from its companion. Common envelope evolution presumably occurred to form the $\mathrm{sdB}$ star while the companion was still on the main sequence, the latter losing insufficient envelope mass in the process to substantially alter its future evolution. On leaving the main sequence, the companion expanded to fill its Roche Lobe, giving the system presently observed.

Table 4. Interstellar and derived atmospheric parameters. Subscripts ' $s$ ' and 'c' refer, respectively, to the hot subdwarf and cool companion (where this was known to exist and accounted for in the fitting). $\alpha$ is the derived stellar angular radius.

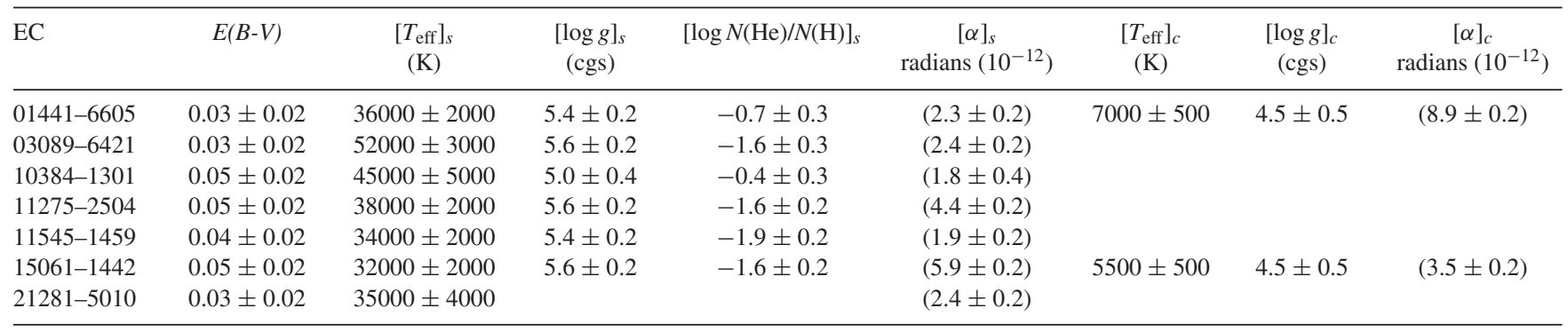


Table 5. Fundamental parameters for hot-subdwarfs.

\begin{tabular}{lcccr}
\hline EC & $\begin{array}{c}\text { Distance } \\
(\mathrm{kpc})\end{array}$ & $R / R_{\odot}$ & $\mathrm{M} / \mathrm{M}_{\odot}$ & $\log _{10} \mathrm{~L} / \mathrm{L}_{\odot}$ \\
\hline $01441-6605$ & $1.594 \pm 0.097$ & $0.163 \pm 0.017$ & $0.24 \pm 0.12$ & $1.624 \pm 0.134$ \\
$03089-6421$ & $0.817 \pm 0.029$ & $0.087 \pm 0.008$ & $0.11 \pm 0.05$ & $1.719 \pm 0.128$ \\
$10384-1301$ & $2.462 \pm 0.661$ & $0.197 \pm 0.057$ & $0.14 \pm 0.15$ & $2.176 \pm 0.318$ \\
$11275-2504$ & $0.778 \pm 0.038$ & $0.152 \pm 0.010$ & $0.33 \pm 0.16$ & $1.658 \pm 0.109$ \\
$11545-1459$ & $2.311 \pm 0.454$ & $0.195 \pm 0.043$ & $0.35 \pm 0.22$ & $1.681 \pm 0.219$ \\
$15061-1442$ & $0.633 \pm 0.027$ & $0.166 \pm 0.009$ & $0.40 \pm 0.19$ & $1.435 \pm 0.119$ \\
$21281-5010$ & $1.459 \pm 0.145$ & $0.155 \pm 0.020$ & & $1.535 \pm 0.229$ \\
\hline
\end{tabular}

Table 6. Fundamental parameters for cool companions.

\begin{tabular}{lccc}
\hline EC & $R / R \odot$ & $\mathrm{M} / \mathrm{M}_{\odot}$ & $\log _{10} \mathrm{~L} / \mathrm{L} \odot$ \\
\hline $01441-6605$ & $0.63 \pm 0.04$ & $0.45 \pm 0.53$ & $-0.05 \pm 0.14$ \\
$15061-1442$ & $0.98 \pm 0.07$ & $1.11 \pm 1.29$ & $-0.08 \pm 0.17$ \\
\hline
\end{tabular}

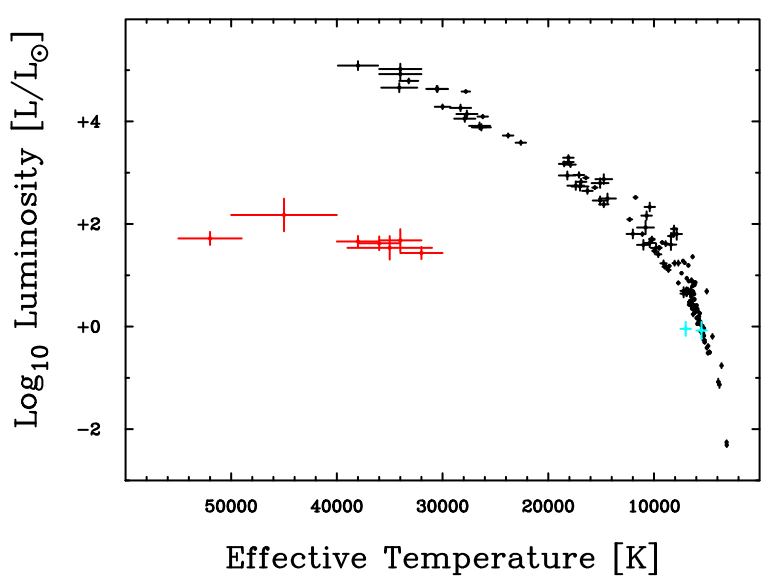

Figure 13. Hertzsprung-Russell diagram for hot subwarfs (red) and their companions (cyan) with main sequence (black) stars being taken from Torres, Andersen \& Giménez (2010).

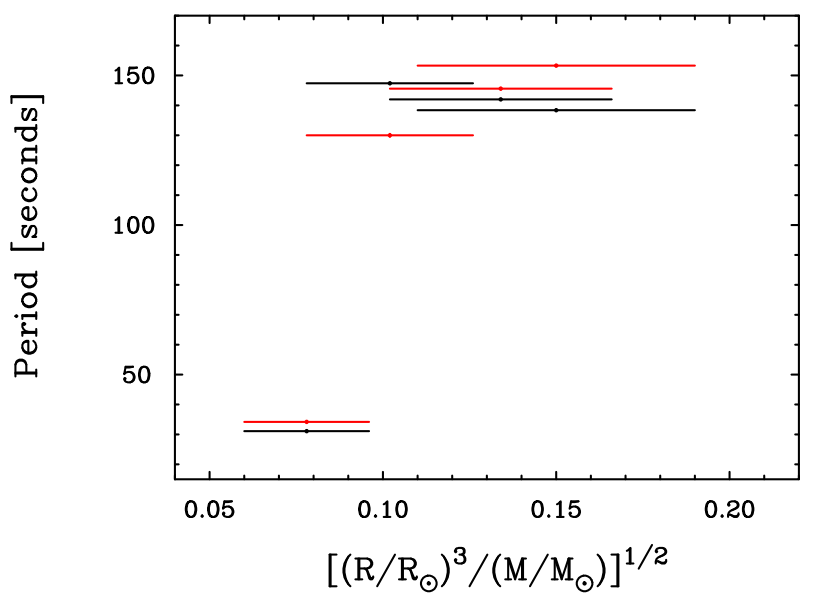

Figure 14. Periods from Table 2 Columns 8 (black) and 11 (red) plotted against a scaled reciprocal square-root of the mean density EC 10384-1301 was excluded on account of its highly uncertain mass determination. We also excluded EC 21281-5010 for which no mass has been determined.
$\Delta$ Magn

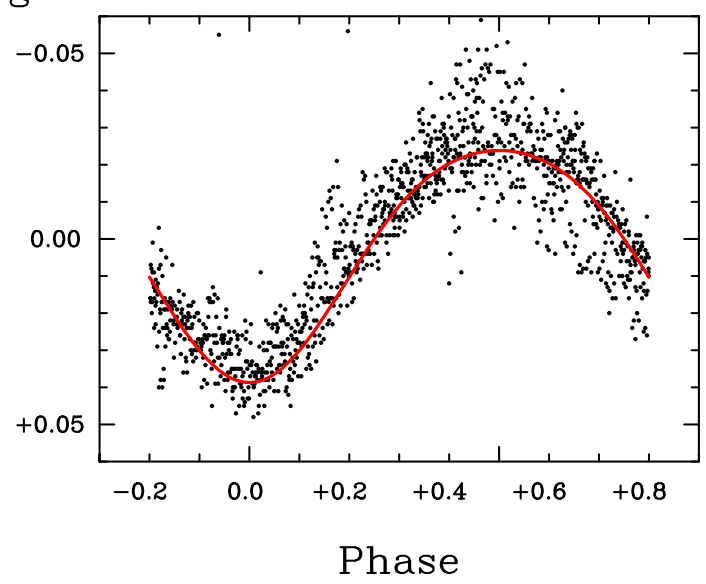

Figure 15. White-light photometry for EC 15061-1442, phased on the assumption that it is a reflection effect binary. The red curve was calculated with the 2010 version of the Wilson-Devinney code as explained in the text

\section{CONCLUDING REMARKS}

Six new hot subdwarf variables have been reported and using available photometry and spectroscopy, we have determined atmospheric parameters. GAIA parallaxes have provided hot subdwarf masses through spectroscopic determinations of surface gravity and photometric measurements of angular radii. Objects of particular interest were then identified.

Vos et al. (2019) study the orbital period-mass ratio relation of wide $\mathrm{sdB}+\mathrm{MS}$ binaries and their probable formation through the Roche Lobe Overflow channel. Wide sdB + MS binaries have periods of $\sim 1000$ days and orbit determination is necessarily more challenging than in the case of close $\mathrm{sdB}+\mathrm{MS}$ binaries. As noted above, EC 01441-6605 after further study could be an addition to the Vos et al. (2019) sample.

EC 03089-6421 was tentatively identified as an ELM WD progenitor and found, through its observed pulsation periods (Fig. 14), to have an internal structure different from that of the other pulsating hot subdwarfs (EC 11275-2504 and EC 11545-1459) for which a comparison could be made. As such EC 03089-6421 could prove to be a critical constraint on ELM WD evolution models, but there is first a requirement for better determinations of $T_{\text {eff }}$ and $\log g$. Metal abundances obtained from ultraviolet spectra will be needed allow line-blanketed non-LTE model atmospheres and synthetic spectra to be computed; these in turn would lead to improved metal abundances and atmospheric parameters, an iterative procedure which would need to be continued to convergence.

Maxted et al. (2002) obtain a reflection-effect light curve and period for PG 1017-086 very similar to those we have obtained 
for EC 15061-1442. Radial velocity data by Maxted et al. (2002) for PG $1017-086$ suggest a smaller mass $\left(0.07 \mathrm{M}_{\odot}\right)$ for the cool companion than we found in the case of EC 15061-1442, giving a wider companion separation and a less highly inclined orbit $\left(\sin i \simeq 63^{\circ}\right)$. If the EC $15061-1442$ binary is as close as is claimed in this paper, with the hot subdwarf accreting mass from its companion, orbital period changes should be observable over a time-scale of years. Nonetheless, the Maxted et al. (2002) study of PG 1017-086 emphasizes the importance of securing radial velocity data for EC 15061-1442 before any firm conclusions may be made.

Systematic errors in our mass determinations arise primarily through the use of model stellar atmospheres where elements other than hydrogen and helium are assumed to be absent; this will affect our angular radii and more importantly our $\log g$ determinations, although the consequences are minimized by fitting line wings only. But the canonical sdB star mass against which we have compared our mass estimates is also compromised by an unknown systematic error. Modelling sdB progenitor (red giant) evolution, where convection is treated by the mixing length theory (BöhmVitense 1958; Arnett et al. 2015), and where angular momentum coupling within the envelope is not well-understood (Eggenberger et al. 2017) are two examples which translate to an uncertain canonical sdB star mass. Opacities used in contemporary stellar evolution calculations also need revision as Lynas-Gray et al. (2018) discuss. Further comparisons between sdB star masses and their canonical value may be expected to contribute to future model improvements.

An obvious improvement, applicable to all stars studied, would be to base a future analysis on higher resolution and better signal-tonoise spectra. Reed et al. (2019) publish an elaborate study, parallel to ours in several respects, of PG $0048+091$ and PG 1315-123 and demonstrate what is achievable given high-quality data and models. Furthermore, Reed et al. (2019) use the EMCEE Monte Carlo Markov Chain sampler (Foreman-Mackey et al. 2013) robustly to find the best-fitting model, along with the corresponding parameters and associated errors.

\section{ACKNOWLEDGEMENTS}

This paper uses observations made at the South African Astronomical Observatory; is based partly upon work supported financially by the University of the Western Cape and the National Research Foundation of South Africa; and has made use of the SIMBAD data base operated at the Centre de Données astronomiques de Strasbourg (CDS) in Strasbourg, France. We are grateful to the referee, Prof. M.D. Reed, for comments which have enabled the authors to improve this paper.

\section{REFERENCES}

Ahmad A., Jeffery C. S., 2005, A\&A, 437, L51

Amôres E. B., Lépine J. R. D., 2005, AJ, 130, 659

Arnett W. D., Meakin C., Viallet M., Campbell S. W., Lattanzio J. C., Mocak M., 2015, ApJ, 809, 20

Bailer-Jones C. A. L., Rybizki J., Fouesneau M., Mantelet G., Andrae R., 2018, AJ, 156, 58

Baran A. S. et al., 2018, MNRAS, 481, 2721

Barlow B. N., Dunlap B. H., Clemens J. C., Reichart D. E., Ivarsen K. M., Lacluyze A. P., Haislip J. B., Nysewander M. C., 2011, MNRAS, 414, 3434

Beers T. C., Preston G. W., Schectman S. A., 1992, AJ, 103, 1987

Bessell M. S., Castelli F., Plez B., 1998, A\&A, 333, 231

Bianchi L., Shiao B., Thilker D., 2017, ApJS, 230, 24
Bohlin R. C., Gilliland R. L., 2004, AJ, 128, 3053

Böhm-Vitense E., 1958, Zeitschrift für Astrophysik, 46, 108

Borucki W. J. et al., 2010, Science, 327, 977

Charpinet S., Van Grootel V., Reese D., Fontaine G., Green E. M., Brassard P., Chayer P., 2008, A\&A, 489, 377

Charpinet et al., 2011, Nature, 480, 496

Cohen M., Wheaton W. A., Megeath S. T., 2003, AJ, 126, 1090

Coppejans R. et al., 2013, PASP, 125, 976

Deeming T. J., 1968, Vistas Astron., 10, 125

Deeming T. J., 1975, Ap\&SS, 36, 137

Drake A. J. et al., 2009, ApJ, 696, 870

Dworetsky M. M., 1983, MNRAS, 203, 917

Eddington A. S., 1918, MNRAS, 79, 2

Eddington A. S., 1919, MNRAS, 79, 171

Eggenberger P. et al., 2017, A\&A, 599, 18

Evans D. W. et al., 2018, A\&A, 616, 21

Fontaine G., Brassard P., Charpinet S., Green E. M., Randall S. K., Van Grootel V., 2012, A\&A, 539, 8

Foreman-Mackey D., Hogg D. W., Lang D., Goodman J., 2013, PASP, 125, 306

Foster H. M., Reed M. D., Telting J. H., Østensen R. H., Baran A. S., 2015, ApJ, 805, 94

Green E. M. et al., 2003, ApJ, 583, L31

Heber U., 2016, PASP, 128, 966

Hill G., 1982, Publ. Dom. Astrophys. Obs., 16, 67

Husser T.-O., Wende-von Berg S., Dreizler S., Homeier D., Reiners A., Barman T., Hauschildt P. H., 2013, A\&A, 553, 9

Hutchens Z. L., Barlow B. N., Soto A. V., Reichart D. E., Haislip J. B., Kouprianov V. V., Linder T. R., Moore J. P., 2017, Open Astron., 26, 252

Kern J. W., Reed M. D., Baran A. S., Østensen R. H., Telting J. H., 2017, MNRAS, 465, 1057

Kilkenny D., 2010, Astrophys. Space. Sci., 329, 175

Kilkenny D., Koen C., O'Donoghue D., Stobie R. S., 1997a, MNRAS, 285, 640

Kilkenny D., O’Donoghue D., Koen C., Stobie R. S., Chen A., 1997b, MNRAS, 287, 867

Kilkenny D., O’Donoghue D., Worters H. L., Koen C., Hambly N., MacGillivray H., 2015, MNRAS, 453, 1879

Kilkenny D., Worters H. L., O'Donoghue D., Koen C., Koen T., Hambly N., MacGillivray H., Stobie R. S., 2016, MNRAS, 459, 4343

Kilkenny D., Worters H. L., Østensen R. H., 2017, MNRAS, 467, 3963

Koen C., O’Donoghue D., Kilkenny D., Lynas-Gray A. E., Marang F., van Wyk F., 1998, MNRAS, 296, 317

Kurtz D. W., 1985, MNRAS, 213, 773

Latour M., Fontaine G., Brassard P., Green E. M., Chayer P., Randall S. K., 2011, ApJ, 733, 100

Latour M., Randall S. K., Fontaine G., Bono G., Calamida A., Brassard P., 2014, ApJ, 795, 106

Li Z., Chen X., Chen H.-L., Han Z., 2019, ApJ, 871, 148

Lutz R., Schuh S., Silvotti R., 2012, Astron. Nachr., 333, 1099

Lynas-Gray A. E., Basu S., Bautista M. A., Colgan J., Mendoza C., Tennyson J., Trampedach R., Turck-Chieze S., 2018, in Mendoza C., Turck-Chièze S., Colgan J., eds, ASP Conf. Ser. Vol 515, Workshop on Astrophysical Opacities. Astron. Soc. Pac., San Francisco, p. 301

Maxted P. F. L., Marsh T. L., Heber U., Morales-Rueda L., North R. C., Lawson W. A., 2002, MNRAS, 333, 231

Naslim N., Jeffery C. S., Behara N. T., Hibbert A., 2011, MNRAS, 412, 363

Németh P., Østensen R., Tremblay P., Hubeny I., 2014, in van Grootel V., Green E., Fontaine G., Charpinet S., eds, ASP Conf. Ser. Vol. 481, 6th Meeting on Hot Subdwarf Stars and Related Objects. Astron. Soc. Pac., San Francisco, p. 95

Norris J. E., Ryan S. G., Beers T. C., 1999, ApJS, 123, 639

O’Donoghue D., Kilkenny D., Koen C., Hambly N., MacGillivray H., Stobie R. S., 2013, MNRAS, 431, 240

Oreiro R., Ulla A., Pérez Hernández F., Østensen R., Rodriguez López C., MacDonald J., 2004, A\&A, 418, 243 
Østensen R. H., Reed M. D., Baran A. S., Telting J. H., 2014, A\&A, 564, 4

Otani T., Oswalt T. D., Lynas-Gray A. E., Kilkenny D., Koen C., Amaral M., Jordan R., 2018, ApJ, 859, 13

Pablo H. et al., 2012, MNRAS, 422, 1343

Preece H. P., Tout C. A., Jeffery C. S., 2018, MNRAS, 481, 715

Randall S. K., Calamida A., Fontaine G., Bono G., Brassard P., 2011, ApJ, 737,4

Randall S. K. et al., 2016, A\&A, 589, 17

Reed M. D., Foster H., Telting J. H., Østensen R. H., Farris L. H., Oreiro R., Baran A. S., 2014, MNRAS, 440, 3809

Reed M. D. et al., 2018, Open Astron., 27, 157

Reed M. D. et al., 2019, MNRAS, 483, 2282

Remie H., Lamers H. J. G. L. M., 1982, A\&A, 105, 85

Ricker G. R. et al., 2015, J. Astron. Telesc. Instrum. Syst., 1, 014003

Schechter P. L., Mateo M., Saha A., 1993, PASP, 105, 1342

Schindler J.-T., Green E. M., Arnett W. D., 2015, ApJ, 806, 178

Schuh S., Huber J., Dreizler S., Heber U., O'Toole S. J., Green E. M., Fontaine G., 2006, A\&A, 445, L31

Silvotti R. et al., 2007, Nature, 449, 189
Skrutskie M. F. et al., 2006, AJ, 131, 1163

Stobie R. S. et al., 1997, MNRAS, 287, 848

Stroeer A., Heber U., Lisker T., Napiwotzki R., Dreizler S., Christlieb N., Riemers D., 2007, A\&A, 462, 269

Telting J. H. et al., 2012, A\&A, 544, 16

Torres G., Andersen J., Giménez A., 2010, Astron. Astrophys. Rev., 18, 67

Van Grootel V., Charpinet S., Fontaine G., Brassard P., Green E. M., Chayer P., Randall S. K., 2008, A\&A, 488, 685

Vos J., Vučković M., Chen X., Han Z., Boudreaux T., Barlow B. N., Østensen R., Németh P., 2019, MNRAS, 482, 4592

Weiler M., 2018, A\&A, 617, 15

Wilson R. E., 1990, ApJ, 356, 613

Wilson R. E., 2008, ApJ, 672, 575

Wilson R. E., Devinney E. J., 1971, ApJ, 166, 605

Woudt P. A. et al., 2006, MNRAS, 371, 1497

Wright E. L. et al., 2010, AJ, 140, 1868

This paper has been typeset from a $\mathrm{T}_{\mathrm{E}} \mathrm{X} / \mathrm{L} \mathrm{T} \mathrm{E}$ file prepared by the author. 